\title{
SPACES OF MAPPINGS ON TOPOLOGICAL PRODUCTS WITH APPLICATIONS TO HOMOTOPY THEORY
}

JAMES R. JACKSON

1. Introduction. If $A$ and $B$ are arbitrary topological spaces, we denote by $B^{A}$ the compact-open topologized space of continuous functions on $A$ into $B$. This topology is defined by taking as a subbase the collection of sets

$$
(K, W)=\left\{f \mid f \in B^{A}, f(K) \subset W\right\},
$$

for $K$ a compact subset of $A$ and $W$ an open subset of $B[2]^{1}$.

Now let $X$ and $Y$ be Hausdorff spaces, and let $Z$ be an arbitrary topological space. Let $X \times Y$ be the topological product of $X$ and $Y$. We shall be concerned with the compact-open topologized spaces $Z^{X \times Y}, Z^{X}$, and $\left(Z^{X}\right)^{Y}$. R. H. Fox [2] has related these spaces, taking $Z^{X}$ as compact-open topologized, but not considering topologies on the more complex spaces. Our basic result is the following theorem, which, with its corollary, adds significant conclusions to the results of Fox.

(1.1) Theorem. For $f \in Z^{X \times Y}$, define $\sigma(f): Y \rightarrow Z^{X}$ by

$$
\sigma(f)(y)(x)=f(x, y) \quad x \in X, y \in Y .
$$

Then $\sigma$ is a homeomorphism of $Z^{X \times Y}$ into $\left(Z^{X}\right)^{Y}$.

We defer the proof of (1.1) until $\$ \S 2$ and 3. In the meantime we state and prove a corollary which will be useful in the sequel.

(1.3) CoRollary. If either (a) $X$ and $Y$ are both locally separable, or (b) $X$ is regular and locally compact; then

$$
\sigma: Z^{X \times Y} \rightarrow\left(Z^{X}\right)^{Y}
$$

is a homeomorphism onto.

According to the theorem, we need only show that $\sigma$ is onto. But by a result of Fox [2], (1.2) (essentially) defines a transformation of $\left(Z^{X}\right)^{Y}$ into $Z^{X \times Y}$ if either (a) or (b) holds. From this the required condition is obvious.

2. An important lemma. The following lemma, which is of some interest in itself, is essential to our proof ( $\$ 3)$ of the continuity of $\sigma$.

Received by the editors July 22, 1951.

${ }^{1}$ Numbers in brackets refer to the bibliography at the end of the paper. 
(2.1) Lemma. Let $A$ be a Hausdorff space, and let $\{U\}$ be a subbase for the topology on $B$. Then the collection of sets $(K, U)$, for $K a$ compact subset of $A$ and $U \in\{U\}$, is a sub-base for the compact-open topology on $B^{A}$.

Proof. It is plainly enough to show that if $K$ is a compact subset of $A$ and $W$ is an open subset of $B$, and if $f \in(K, W)$, then there exist compact subsets $K_{1}, \cdots, K_{m}$ of $A$, and sets $U_{1}, \cdots, U_{m} \in\{U\}$, such that

$$
f \in \bigcap_{i=1}^{m}\left(K_{i}, U_{i}\right) \subset(K, W) .
$$

Since $W$ is open in $B$, we can write

$$
W=\bigcup_{\alpha \in A}\left[\bigcap_{i=1}^{n_{\alpha}} U_{i}^{\alpha}\right],
$$

where each $U_{1}^{\alpha} \in\{U\}$. For each $x \in K, f(x)$ is in some $\bigcap_{t=1}^{n_{\alpha}} U_{1}^{\alpha}$, which we denote by $\bigcap_{i=1}^{n_{x}} U_{i}^{x}$. Since $f$ is continuous, each $x$ has a neighborhood $V_{0}^{x} i n^{2} K$ such that

$$
f\left(V_{0}^{x}\right) \subset \bigcap_{i=1}^{n_{x}} U_{i}^{x}
$$

As a compact Hausdorff space, $K$ is regular, so that each $x$ has a neighborhood $V^{x}$ in $K$, whose closure in $K, K^{x}$, is contained in $V_{0}^{x}$.

The collection $\left\{V^{x} \mid x \in K\right\}$ is an open covering of the compact space $K$, and hence has a finite subcovering $\left\{V_{j} \mid j=1, \cdots, k\right\}$. Denote the $V_{0}^{x}, \bigcap_{i=1}^{n_{x}} U_{i}^{x}$, and $K^{x}$ corresponding to $V_{j}$ by $V_{0}^{j}, \bigcap_{i=1}^{n_{i}} U_{i}^{j}$, and $K_{j}$, respectively.

Now the sets $K_{j}$, as closed subsets of compact set $K$, are compact. Moreover,

$$
f\left(K_{j}\right) \subset f\left(V_{0}^{j}\right) \subset \bigcap_{i=1}^{n_{j}} U_{i}^{j}, \quad j=1, \cdots, k .
$$

Hence,

$$
f \in \bigcap_{j=1}^{k}\left[\bigcap_{i=1}^{n_{j}}\left(K_{j}, U_{i}^{j}\right)\right] .
$$

Suppose $g$ is in the set on the right-hand side of (2.3). If $x \in K$, then

2 A neighborhood of $x$ in $K$ is a set containing $x$, and open as a subset of the subspace $K$. 
$x$ is in some $V_{j}$, and hence is in $K_{j}$. Therefore, $g(x) \in \bigcap_{i=1}^{n_{j}} U_{i}^{j}$, so that $g(x) \in W$. Thus $g \in(K, W)$, so that

$$
\bigcap_{j=1}^{k}\left[\bigcap_{i=1}^{n_{j}}\left(K_{j}, U_{i}^{j}\right)\right] \subset(K, W) .
$$

Together, (2.3) and (2.4) show that (2.2) is satisfied, so the lemma is proved.

3. Proof of (1.1). Fox [2] shows without restriction that for each $f, \sigma(f) \in\left(Z^{X}\right)^{Y}$. That $\sigma$ carries $Z^{X \times Y}$ onto $\sigma\left(Z^{X \times Y}\right)$ in a one-one fashion is obvious. Hence it remains to prove that $\sigma$ is continuous, and that $\sigma^{-1}$ is continuous on $\sigma\left(Z^{X \times Y}\right)$.

Since $Y$ is a Hausdorff space, and since the sets $(K, W)$, for $K$ a compact subset of $X$ and $W$ an open subset of $Z$, form a sub-base for $Z^{X}$, it follows from $(2.1)$ that the subsets $(L,(K, W))$ of $\left(Z^{X}\right)^{Y}$ form a sub-base for this space, where $K$ runs through the compact subsets of $X, L$ runs through the compact subsets of $Y$, and $W$ runs through the open subsets of $Z$. It is plain that

$$
\sigma^{-1}(L,(K, W))=(K \times L, W) .
$$

Now $K \times L$, as the topological product of compact sets, is compact, so $(K \times L, W)$ is open. It follows easily that the inverse under $\sigma$ of any open subset of $\left(Z^{X}\right)^{Y}$ is open, so $\sigma$ is continuous.

(Note: In the proof that $\sigma$ is continuous, we have used the hypothesis that $Y$ be a Hausdorff space, but not the condition that $X$ be a Hausdorff space.)

It remains to be shown that $\sigma^{-1}$ is continuous on $\sigma\left(Z^{X \times Y}\right)$. For this, it will be sufficient to prove that if $J$ is a compact subset of $X \times Y$, if $W$ is an open subset of $Z$, and if $f \in \sigma(J, W)$, then there exist compact subsets $K_{1}, \cdots, K_{n}$ of $X$, and compact subsets $L_{1}, \cdots, L_{n}$ of $Y$, such that

$$
f \in \sigma\left(Z^{X \times Y}\right) \cap\left[\bigcap_{i=1}^{n}\left(L_{i},\left(K_{i}, W\right)\right)\right] \subset \sigma(J, W) .
$$

For then $\sigma(J, W)$ must be open in $\sigma\left(Z^{X \times Y}\right)$, as required.

Let $f=\sigma\left(f_{0}\right)$, where $f_{0} \in(J, W)$. Let $J_{X}$ and $J_{Y}$ be the projections of $J$ in $X$ and $Y$, respectively. For $(x, y) \in J$, pick neighborhoods $U_{0}^{x, y}$ of $x$ in $J_{X}$ and $V_{0}^{x, y}$ of $y$ in $J_{Y}$, such that

$$
f_{0}\left(U_{0}^{x, y} \times V_{0}^{x, y}\right) \subset W .
$$

This is possible since $f_{0}$ is continuous. As in the proof of (2.1), pick 
neighborhoods $U^{x, y}$ of $x$ in $J_{X}$ and $V^{x, y}$ of $y$ in $J_{Y}$, such that the closure $K^{x, y}$ of $U^{x, y}$ in $J_{X}$ is contained in $U_{0}^{x, y}$, and the closure $L^{x, y}$ of $V^{x, v}$ in $J_{Y}$ is contained in $V_{0}^{x, y}$.

The collection $\left\{\left(U^{x, y} \times V^{x, y}\right) \cap J \mid(x, y) \in J\right\}$ is an open covering of the compact space $J$, and hence has a finite subcovering $\left\{\left(U^{i} \times V^{i}\right)\right.$ $\cap J \mid i=1, \cdots, n\}$. Denote the $U_{0}^{x, y}$ and the $K^{x, y}$ corresponding to $U^{i}$ by $U_{0}^{i}$ and $K_{i}$, respectively, and the $V_{0}^{x, y}$ and $L^{x, y}$ corresponding to $V^{i}$ by $V_{0}^{i}$ and $L_{i}$.

The $K_{i}$ and $L_{i}$, as closed subsets of compact sets, are themselves compact. Moreover,

$$
f\left(L_{i}\right)\left(K_{i}\right)=f_{0}\left(K_{i} \times L_{i}\right) \subset f_{0}\left(U_{0}^{i} \times V_{0}^{i}\right) \subset W,
$$

for $i=1, \cdots, n$. Hence

$$
f \in \sigma\left(Z^{X \times Y}\right) \cap\left[\bigcap_{i=1}^{n}\left(L_{i},\left(K_{i}, W\right)\right)\right] .
$$

Suppose $g$ is in the set on the right side of (3.2). Let $g=\sigma\left(g_{0}\right)$, where $g_{0} \in Z^{\mathbf{x} \times Y}$. Then

$$
g_{0}\left(K_{i} \times L_{i}\right)=g\left(L_{i}\right)\left(K_{i}\right) \subset W, \quad i=1, \cdots, n .
$$

If $(x, y) \in J$, then $(x, y)$ is in some $U^{i} \times V^{i}$, and hence is in $K^{i} \times L^{i}$. Hence $g_{0} \in(J, W)$, so $g \in \sigma(J, W)$. Thus,

$$
\sigma\left(Z^{X \times Y}\right) \cap\left[\bigcap_{i=1}^{n}\left(L_{i},\left(K_{i}, W\right)\right)\right] \subset \sigma(J, W) .
$$

By (3.2) and (3.3), (3.1) is satisfied, as required.

4. Notation. If $A$ and $B$ are topological spaces, and $A_{1}, \cdots, A_{n}$ are subsets of $A$, while $B_{1}, \cdots, B_{n}$ are subsets of $B$, then we shall denote by $B^{A}\left\{A_{1}, B_{1} ; \cdots ; A_{n}, B_{n}\right\}$ the subspace of $B^{A}$ consisting of functions $f$ with

$$
f\left(A_{i}\right) \subset B_{i}, \quad i=1, \cdots, n .
$$

If $b_{0}$ is any point, then the same symbol $b_{0}$ will be used to denote both the set whose only member is $b_{0}$, and any constantly $b_{0}$-valued function.

If $B$ is any space (possibly a function space), and $b_{0} \in B$, then $\Pi_{m}\left(B, b_{0}\right)$ denotes the $m$ th homotopy group of $B$ at base-point $b_{0}$. If $B_{0}$ is a subset of $B$ with $b_{0} \in B_{0}$, then $\Pi_{m}\left(B, B_{0}, b_{0}\right)$ is the $m$ th relative homotopy group of $B$ relative to $B_{0}$ at base-point $b_{0}[1]$.

If there exists a homomorphism of the group $G$ onto the subgroup 
$H$, which is the identity over $H$, and whose kernel is $N$, then $G$ is called a split extension of $N$ by $H$. Then the structure of $G$ is determined by the structures of $N$ and $H$ and the commutators $[n, h]$ for $n \in N, h \in H$. If $H$ is a normal subgroup, then $G$ is the direct sum $N+H[1]$.

In the following sections, $I$ will denote the closed unit interval, $S$ the circle, $S^{0}$ will be a pair of points, $S^{r}$ the $r$-sphere $(r>0)$, and $s_{r}$ will be a point of $S^{r}(r \geqq 0)$. $T^{0}$ will denote a single point, and $T^{r}$ will be the $r$-fold topological product of 1-spheres $(r>0)$.

5. Torus homotopy groups. Let $Y$ be a topological space, and let $y_{0} \in Y_{0} \subset Y$. Define

$$
\begin{array}{r}
\tau_{r}^{m}\left(Y, Y_{0}, y_{0}\right)=\Pi_{m}\left(Y^{T^{r-2} \times I}\left\{T^{r-2} \times 0, Y_{0} ; T^{r-2} \times 1, y_{0}\right\}, y_{0}\right), \\
r=2,3, \cdots ; m=1,2, \cdots .
\end{array}
$$

Fox [1] observed that $\tau_{r}^{1}\left(Y, Y_{0}, y_{0}\right)$ is isomorphic to his $r$ th relative torus homotopy group $\tau_{r}\left(Y, Y_{0}, y_{0}\right)$. He proved a structure theorem for his torus homotopy groups $\tau_{r}\left(Y, y_{0}\right)[1,9.3]$, using a laborious construction of successive homomorphisms. This theorem is easily proved by use of (1.3). Rather than state and prove this published result, we give a (slight generalization of a) corresponding theorem concerning the relative torus groups.

(5.2) Theorem. (i) $\tau_{2}^{m}\left(Y, Y_{0}, y_{0}\right)$ is isomorphic to $\Pi_{m+1}\left(Y, Y_{0}, y_{0}\right)$.

(ii) If $\tau_{r}^{m}\left(Y, Y_{0}, y_{0}\right)$ is abelian (in particular, if $\left.m>1\right)$, then it is isomorphic to the direct sum ${ }^{3}$

$$
\sum_{j=2}^{r} C_{r-2, j-2} \Pi_{m+j-1}\left(Y, Y_{0}, y_{0}\right) \text {. }
$$

(iii) If $r>2$, then $\tau_{r}^{m}\left(Y, Y_{0}, y_{0}\right)$ is a split extension of a subgroup isomorphic to $\tau_{r-1}^{m+1}\left(Y, Y_{0}, y_{0}\right)$ by a subgroup isomorphic to $\tau_{r-1}^{m}\left(Y, Y_{0}, y_{0}\right)$.

Proof. Conclusion (i) was noted by Fox. Conclusion (ii) is a simple inductive consequence of the case $r=2$ (contained in (i)) and conclusion (iii). Thus, it remains to prove (iii).

It is obvious from (1.3) that there is a homeomorphism of

$$
Y^{T^{r-2} \times I}\left\{T^{r-2} \times 0, Y_{0} ; T^{r-2} \times 1, y_{0}\right\}
$$

onto

$$
\left(Y^{I}\left\{0, Y_{0} ; 1, y_{0}\right\}\right)^{T^{r-2}},
$$

8 The $C_{j, k}$ are the binomial coefficients. 
and a homeomorphism of this space onto

$$
\left[\left(Y^{I}\left\{0, Y_{0} ; 1, y_{0}\right\}\right)^{T^{r-3}}\right]^{S},
$$

and that the functions $y_{0}$ of these various spaces correspond under the homeomorphisms. (Note that the elements of the last space are "third order" functions.)

According to S. T. Hu [3], $\Pi_{m}\left(B^{S}, b_{0}\right)$ is a split extension of a subgroup isomorphic to $\Pi_{m+1}\left(B, b_{0}\right)$ by a subgroup isomorphic to $\Pi_{m}\left(B, b_{0}\right)$. Then since $\tau_{r}^{m}\left(Y, Y_{0}, y_{0}\right)$ is isomorphic to the $m$ th homotopy group of the space given by (5.3), at base-point $y_{0}$, we may conclude that $\tau_{r}^{m}\left(Y, Y_{0}, y_{0}\right)$ is a split extension of a subgroup isomorphic to

$$
\Pi_{m+1}\left(\left(Y^{I}\left\{0, Y_{0} ; 1, y_{0}\right\}\right)^{T^{r-3}}, y_{0}\right)
$$

by a subgroup isomorphic to

$$
\Pi_{m}\left(\left(Y^{I}\left\{0, Y_{0} ; 1, y_{0}\right\}\right)^{T^{r-3}}, y_{0}\right) .
$$

Applying (1.3) again, we see that the groups (5.4) and (5.5) are isomorphic to $\tau_{r-1}^{m+1}\left(Y, Y_{0}, y_{0}\right)$ and $\tau_{r-1}^{m}\left(Y, Y_{0}, y_{0}\right)$, respectively. This completes the proof.

6. Further applications. Most known theorems on homotopy groups of function spaces can be combined with (1.3) to yield additional results on homotopy groups, and, often, to get analogous theorems concerning certain relative homotopy groups, torus homotopy groups, and relative torus homotopy groups of function spaces. Using " $\simeq$ " to indicate algebraic isomorphism, and $G \simeq N^{\prime} \otimes H^{\prime}$ to mean that $G$ is a split extension of a subgroup isomorphic to $N^{\prime}$ by a subgroup isomorphic to $H^{\prime}$, we state several interesting results which follow from (1.3) and the theorems of [3], in much the same way that we obtained (5.2). We include the known results (6.1) and (6.5) for completeness. In (6.2), (6.4), (6.6), and (6.8), the second function space involved is a subspace of the first in the obvious way.

$$
\begin{aligned}
& \Pi_{m}\left(Y^{S^{k}}\left\{s_{k}, y_{0}\right\}, y_{0}\right) \simeq \Pi_{m+k}\left(Y, y_{0}\right) . \\
& \Pi_{m}\left(Y^{S^{k}}\left\{s_{k}, y_{0}\right\}, Y_{0}^{S^{k}}\left\{s_{k}, y_{0}\right\}, y_{0}\right) \simeq \Pi_{m+k}\left(Y, Y_{0}, y_{0}\right) . \\
& \tau_{r}^{m}\left(Y{ }^{S^{k}}\left\{s_{k}, y_{0}\right\}, y_{0}\right) \simeq \tau_{r}^{m+k}\left(Y, y_{0}\right) . \\
& \tau_{r}^{m}\left(Y^{S^{k}}\left\{s_{k}, y_{0}\right\}, Y_{0}^{S^{k}}\left\{s_{k}, y_{0}\right\}, y_{0}\right) \simeq \tau_{r}^{m+k}\left(Y, Y_{0}, y_{0}\right) . \\
& \Pi_{m}\left(Y^{S^{k}}, y_{0}\right) \simeq \Pi_{m+k}\left(Y, y_{0}\right) \otimes \Pi_{m}\left(Y, y_{0}\right) . \\
& \Pi_{m}\left(Y^{S^{k}}, Y_{0}^{S^{k}}, y_{0}\right) \simeq \Pi_{m+k}\left(Y, Y_{0}, y_{0}\right) \otimes \Pi_{m}\left(Y, Y_{0}, y_{0}\right) . \\
& \tau_{r}^{m}\left(Y^{S^{k}}, y_{0}\right) \simeq \tau_{r}^{m+k}\left(Y, y_{0}\right) \otimes \tau_{r}^{m}\left(Y, y_{0}\right) .
\end{aligned}
$$




$$
\tau_{r}^{m}\left(Y^{S^{k}}, Y_{0}^{S^{k}}, y_{0}\right) \simeq \tau_{r}^{m+k}\left(Y, Y_{0}, y_{0}\right) \otimes \tau_{r}^{m}\left(Y, Y_{0}, y_{0}\right) .
$$

The isomorphism (6.3) implies, with certain theorems given in [1], the interesting corollary that the Whitehead products of elements of the groups $\Pi_{m}\left(Y^{s^{k}}\left\{s_{k}, y_{0}\right\}, y_{0}\right), k>0, m=1,2, \cdots$, are all trivial. (6.1) and (6.2) show that the entire homotopy sequence may be reduced, one chunk at a time, to low-dimensional segments of homotopy sequences of suitable function spaces.

\section{BIBLIOGRAPHY}

1. R. H. Fox, Homotopy groups and torus homotopy groups, Ann. of Math. vol. 49 (1947) pp. 471-510.

2. On topologies for function spaces, Bull. Amer. Math. Soc. vol. 51 (1945) pp. 429-432.

3. S. T. Hu, On spherical mappings in a metric space, Ann. of Math. vol. 48 (1946) pp. 717-734.

University of California, Los ANgeles

\section{A THEOREM ON INVOLUTORY TRANSFORMATIONS WITHOUT FIXED POINTS}

\section{AMASA FORRESTER}

This note contains a proof of a theorem conjectured by Dr. Preston C. Hammer in his work on outwardly simple line families.

By $E^{n+1}$ we denote the $(n+1)$-dimensional number space of ordered $(n+1)$-tuples of real numbers; if $X=\left(x_{1}, x_{2}, \cdots, x_{n+1}\right), \quad Y$ $=\left(y_{1}, y_{2}, \cdots, y_{n+1}\right)$, and $\alpha, \beta$ are real numbers, we define as usual

$$
\alpha X+\beta Y=\left(\alpha x_{1}+\beta y_{1}, \cdots\right), \quad(X, Y)=\sum_{i=1}^{n+1} x_{i} y_{i} .
$$

By the $n$-sphere $S^{n}$ we mean the set of all $X$ in $E^{n+1}$ such that $(X, X)=1$; a point $X$ is said to be interior to $S^{n}$ if $(X, X)<1$. A continuous map $\phi: S^{n} \rightarrow S^{n}$ of the $n$-sphere into itself is called an involutory transformation if $\phi(\phi(Q))=Q$ for all $Q$ on $S^{n}$.

TheOREM. Let $\phi: S^{n} \rightarrow S^{n}$ be an involutory transformation of $S^{n}$ without fixed point, and let $P$ be a point interior to $S^{n}$. Then there is a point $Q$ on $S^{n}$ such that $P$ lies on the line segment from $Q$ to $\phi(Q)$.

Received by the editors August 6,1951. 